\title{
Germanica
}

\section{Helmut Pillau, Wildwuchs. Eine Jugend inmitten des zerrissenen Berlin}

Berlin, Pro Business, 2015, 371 p.

Andrée Lerousseau

\section{OpenEdition}

\section{Journals}

Édition électronique

URL : http://journals.openedition.org/germanica/3588

DOI : 10.4000/germanica.3588

ISSN : 2107-0784

\section{Éditeur}

Université de Lille

\section{Édition imprimée}

Date de publication : 30 décembre 2016

Pagination : 186-187

ISBN : 9782913857384

ISSN : 0984-2632

\section{Référence électronique}

Andrée Lerousseau, « Helmut Pillau, Wildwuchs. Eine Jugend inmitten des zerrissenen Berlin »,

Germanica [En ligne], 59 | 2016, mis en ligne le 30 décembre 2016, consulté le 08 janvier 2021. URL http://journals.openedition.org/germanica/3588 ; DOI : https://doi.org/10.4000/germanica.3588 


\section{Comptes rendus de lecture}

Martin Luther, Von den Juden und ihren Lügen - neu bearbeitet und kommentiert von Matthias Morgenstern, Wiesbaden, Berlin University Press, 2016.

Depuis octobre 2016 et jusqu'en octobre 2017 est célébré en Allemagne le " jubilé de Luther », qui commémore le $500^{\mathrm{e}}$ anniversaire du placard par Luther, le 31 octobre 1517, de ses 95 thèses sur la porte du château de Wittemberg. Margot Käßmann, ambassadrice de l'Église évangélique pour le jubilé, affirmait dans une contribution à la Frankfurter Allgemeine Zeitung remontant à avril 2013 que ces commémorations devaient « au-delà de la joie devant les acquis de la Réforme, dire les parts d'ombre ${ }^{1}$. La publication, au milieu de l'année 2016, d'une édition savante et critique, accessible au plus grand nombre, du texte violent de Luther, Von den Juden und ihren Lügen, participe de cette volonté tôt exprimée. Ce texte appartient à la dernière période de production de Luther pour laquelle la critique parle de revirement chez le Réformateur : car si les écrits antérieurs portent l'espoir de convaincre les Juifs de se convertir (et donc de ne pas les attaquer), Des Juifs et de leurs mensonges est un texte agressif.

Le travail scrupuleux et brillant de Matthias Morgenstern, Professeur à l'Université de Tübingen, s'articule en trois moments : après un propos introductif court est édité le texte de Luther accompagné d'un appareil scientifique important (abondantes notes de bas de page, glossaire, index

1. - «Es kann kein Reformationsjubiläum geben, das bei aller Freude über die Errungenschaften der Reformation ihre Schattenseiten nicht benennt ». FAZ du 1.4.2013. http://www.faz.net/aktuell/politik/inland/fremde-federn-margot-kaessmann-die-dunkleseite-der-reformation-12131764.html. Consulté le 8 janvier 2017. 
des noms cités, registre des citations bibliques, registre des références talmudiques) ; un appendice de 25 pages intitulé « Considérations sur un document de la honte », enrichi par une bibliographie, clôt cette édition.

Après une courte introduction du Président du Conseil de l'Église protestante d'Allemagne, Heinrich Bedford-Strohm, évoquant un « texte repoussant » dont l'instrumentalisation notamment par les nazis n'excuse en rien le Réformateur, Matthias Morgenstern exprime, dans sa courte présentation, une première consternation : le texte de Luther existe dans des éditions scientifiques récentes en français $(2015)^{2}$, en italien $(2000)^{3}$, en anglais (1971) ${ }^{4}$ - mais la plus récente édition en allemand moderne date de... 1936 et l'éditeur de l'époque, Walter Holstens, saluait alors cet écrit comme celui auquel « Luther devait sa gloire comme antisémite de premier plan » (XIII). Matthias Morgenstern souligne d'ailleurs d'emblée sa dette à la traduction française, qui date de 2015 et que nous devons à Johannes Honigmann, le fils de Barbara Honigmann : c'est en effet une superbe traduction, elle-même introduite et annotée par un universitaire de talent, Pierre Savy, spécialiste des identités juives et chrétiennes au Moyen Âge et à l'époque moderne 5 . À la sortie de cette traduction française, La Vie des Idées a publié sous le titre « Haines de Luther » une recension riche de la jeune historienne Marion Deschamps 6 . De cette consternation exprimée par Matthias Morgenstern est né un double dessein : tout d'abord rendre disponible au public allemand d'aujourd'hui ce texte que la plupart des Luthériens aimeraient faire oublier. Le texte peut donc être lui-même considéré comme une traduction en allemand contemporain, le choix de la traduction est d'ailleurs souvent documenté en notes de bas de page ; l'ajout en outre de titres et de sous-titres permet de mieux s'orienter dans le cheminement de la pensée de Luther : la table des matières fait ainsi état de 8 grands chapitres, avec une introduction et une conclusion, le septième de ces chapitres étant constitué du fameux catalogue des mesures antijuives, que le huitième chapitre répète à l'attention des pasteurs. En second lieu, et c'est là certainement ce qui fait l'originalité de cette édition, la présentation et le commentaire du texte de Luther sont exprimés du point de vue du judaïsme. Car si Matthias Morgenstern est

2. - Martin Luther, Des Juifs et de leurs mensonges, Édition critique traduite de l'allemand par Johannes Honigmann ; Introduction et notes de Pierre Savy, Paris, Honoré Champion, 2015.

3. - Martin Luther, Degli ebrei e delle loro menzogne, traduction d'Adelisa Malena, introduction d'Adriano Prosperi, Turin, Einaudi, 2000.

4. - Martin Luther, On the Jews and Their Lies, dans Luther's Works, volume 47, édité par H.T. Lehmann, traduction de Martin H. Bertram, Philadelphia, 1971.

5. - Voir le site du laboratoire ACP (Analyse Comparée des Pouvoirs) de l'Université Paris-Est-Marne la Vallée : http://acp.u-pem.fr/equipe/pierre-savy/?no_cache=1.

6. - Voir http://www.laviedesidees.fr/Haines-de-Luther.html. 
pasteur depuis 1999 dans le Wurtemberg, il est aussi professeur à l'Institutum Judaicum de l'Université de Tübingen, chercheur en histoire des religions, spécialiste certes du dialogue judéo-chrétien, mais surtout du Talmud de Jérusalem et de la littérature rabbinique. Le texte de Luther est ainsi commenté à l'aide de quelque 1000 notes de bas de page qui ont un triple objet : certaines renvoient au très riche glossaire comprenant des entrées sur des termes hébraïques (« goj », « prière de l'Aleinu »), sur la tradition juive (circoncision, texte biblique et ses personnages tels Job ou Isaïe), sur les reproches antijudaïques (déicide, profanation de l'hostie, " truie juive », meurtre rituel, diabolisation, usure) et sur de grands personnages de l'époque (Sebastian Münster par exemple). D'autres notes renvoient à des écrits, comme à l'édition de Weimar des textes de Luther qui, pour le volume 53 comprenant Von den Juden und ihren Lügen, date de l'année 1920, et avec lequel la traduction nouvelle est confrontée ; à d'autres textes de Luther ; à des références à l'Ancien ou au Nouveau Testament. Matthias Morgenstern utilise enfin ces notes pour suggérer ce qu'aurait pu rétorquer un exégète juif de l'époque, en invoquant des références au Talmud, à la Thora ou des midrashim : ces réponses juives à Luther plongent le lecteur dans l'univers de la disputation judéo-chrétienne moyenâgeuse.

Pour Matthias Morgenstern, le jubilé de Luther oblige les Allemands à se confronter aux propos de Luther sur les Juifs, à « vraiment tout mettre sur la table »(252). Matthias Morgenstern souligne que les propos haineux sont introduits par un long préambule exégétique déterminant pour Luther qui veut démontrer l'ancrage du mensonge des Juifs dans leur lecture même de la Bible. Le titre même du texte est une référence au verset 8,44 de l'Évangile de Jean dans lequel les deux thèmes $\mathrm{du}$ mensonge et de l'origine diabolique sont liés. Malgré les propos haineux et insupportables, il fallait une publication nouvelle de ce texte dont Matthias Morgenstern rappelle « la coresponsabilité pour les actes de violence et les débordements antijuifs de sa propre époque » (272), mais aussi son utilisation sous le nazisme qui fit dire à Julius Streicher, l'éditeur du Stürmer, lors du procès de Nuremberg, que les juges se trompaient et devraient faire comparaître Luther à sa place. Aussi ne peut-on que saluer cette nouvelle édition commentée avec précision et sans concession en cette année Luther.

Martine Benoit 
Dorothea Bohnekamp (dir.), Penser les identités juives dans l'espace germanique -XIXe-XXe siècles, Presses Universitaires de Rennes, 2015, $176 \mathrm{p}$.

Particulièrement stimulant, cet ouvrage témoigne à double titre, dans un souci constant d'historicité, de la vitalité des études judéo-allemandes dont Dominique Bourel trace l'historique et esquisse les perspectives dans sa préface. Ainsi que le souligne Dorothea Bohnekamp dans son introduction qui est un rappel des " différentes stratégies identitaires mises en place dans le passé pour assurer la pérennité du judaïsme» et pose en outre la question de la définition d'une identité juive " postmoderne ", cette publication allie en effet la synthèse des recherches effectuées par les différents contributeurs et des " approches novatrices », offrant un panorama de l'évolution de la conscience juive, appréhendée à partir d'un certain nombre de paradigmes (le libéralisme, la modernité, la ville), et de ses mutations au cours d'une histoire marquée par une tension permanente entre assimilation et dissimilation et ponctuée de crises identitaires face à l'exacerbation de l'antisémitisme, aux persécutions et au malaise inhérent à la condition juive en Allemagne au lendemain de la Shoah, dans un espace où se côtoient sans nécessairement se rejoindre différentes représentations mémorielles.

La première partie traite de la tradition judéo-allemande qui s'élabore à partir du siècle des Lumières et durant la période de l'émancipation. Fondée sur une adhésion aux idéologies de progrès et sur une intégration et une pleine contribution à la vie culturelle et économique de l'espace germanique, elle va de pair, malgré le danger d'une acculturation, avec un attachement à un judaïsme porteur d'universalisme et ouvert à la modernité. Particulièrement féconde, cette tradition était historiquement vouée à l'échec comme le démontrent Daniel Azuélos et Jacques Le Rider dans une brillante synthèse de leurs études dédiées respectivement aux Juifs allemands et viennois au XIX ${ }^{\mathrm{e}}$ siècle et durant la première moitié $\mathrm{du} \mathrm{Xx}^{\mathrm{e}}$ siècle. Elle devait donner naissance à une forme spécifique de la conscience juive distincte de celle que développèrent les Juifs de l'Est dans un environnement d'emblée hostile, ainsi que l'illustre l'article d'Olivier Baisez effectuant à travers l'exemple de Franz Oppenheimer une lecture de l'originalité du sionisme allemand. La marginalisation progressive des Juifs et le désastre qui s'ensuivit intensifièrent un questionnement toujours présent - que l'on songe à Heine - relatif à une identité plurielle contraire aux aspirations nationalistes, ethniques et totalitaires de la société environnante. Ces « représentations croisées des identités judéo-allemandes », qui ont toutes partie liée avec l'écoute fondatrice de l'identité d'Israël, font l'objet de la seconde partie. Consacrée aux réflexions et aux pratiques des rédacteurs de l'Allgemeine Zeitung des Judentums et des Archives israélites en vue de 
promouvoir un dialogue entre les peuples dans un contexte de guerres et d'animosité, l'analyse de Heidi Knörzer attire l'attention sur le rôle joué par le double héritage juif du messianisme et du cosmopolitisme dans un projet visant à poser les conditions de la « véritable civilisation », fondée sur l'échange. La rencontre est également au cœur de l'exposé de Martine Benoit qui, à travers les représentations de l'« Ostjude » dans la littérature de langue allemande des $\mathrm{XIX}^{\mathrm{e}}$ et $\mathrm{XX}^{\mathrm{e}}$ siècles, retrace les trois grands moments de la découverte et de l'appréhension des Juifs de l'Est, témoignant de l'infléchissement du regard porté sur le judaïsme oriental et de son importance pour la redéfinition d'une identité en péril et pour la préservation de la mémoire. Une même interrogation traverse l'œuvre de Gertrud Kolmar dont Miriam Freitag, dans une analyse fine et ciblée, explore la dialectique subtile entre Ouest et Est. La troisième partie, quant à elle, s'articule autour du paradigme urbain que Joachim Schlör invite à approfondir dans une perspective résolument pluridisciplinaire, mettant l'accent sur l'expérience de la spatialité dans le judaïsme trop systématiquement associé à la seule notion de temporalité. Un bon exemple des recherches qu'il entend promouvoir dans le cadre de son projet autour des Topographies juives est fourni par l'article de Laurence Guillon-Duchaine consacré à la reconstruction de la vie juive à Berlin de 1945 à la réunification et au réinvestissement de l'espace berlinois par une communauté juive hétérogène, contribution que vient compléter celle de Sophie Zimmer s'attachant à décrire le renouveau juif à Berlin, de la réunification à nos jours, renaissance placée sous le signe de la pluralité et de la diversité, caractéristiques communes au judaïsme berlinois et à la ville tout entière.

$\mathrm{Au}$ fil des pages se dessine, ainsi que le mentionne Dorothea Bohnekamp, un "paradigme identitaire unique », une « forme d'hybridation culturelle » aux déclinaisons multiples, qui nous incite à reconsidérer la mémoire et le passé allemands à partir de ce « lieu interstitiel », mais a également valeur de modèle pour penser l'avenir d'une Europe dont la réalité actuelle peine à rejoindre l'idéal d'une unité dans une prise en compte de la diversité. En cette époque de recrudescence de l'antisémitisme et des violences antisémites, où la haine et le mépris des Juifs s'affichent impunément sur le net, il y a urgence à rappeler, à l'instar de cet ouvrage, et à revendiquer haut et fort cet héritage juif que d'aucuns, aujourd'hui comme hier, s'entendent à passer sous silence, voire à éradiquer. « Penser les identités juives dans l'espace germanique », c'est aussi réfléchir à notre identité propre dans l'espace européen. 
Helmut Pillau, Wildwuchs. Eine Jugend inmitten des zerrissenen Berlin, Berlin, Pro Business, 2015, 371 p.

On se plonge avec intérêt dans le récit de cette jeunesse et c'est avec un plaisir constant dû à la qualité de la langue et à un humour et une ironie subtile, qui ne bascule jamais dans la satire, que l'on parcourt ce roman de formation d'un intellectuel quelque peu atypique qui, à partir de 1980, enseignera la littérature comparée à l'Université de Mayence, lorsque sera venu le temps d'un «embourgeoisement » considéré avec une distance critique et amusée par ce Berlinois d'origine et de cœur.

« Écrire sur soi signifiait en même temps écrire sur Berlin », confie Helmut Pillau, et son autobiographie se lit également comme une passionnante chronique de la vie politico-intellectuelle berlinoise, de la fin de la Seconde Guerre mondiale au tout début des années 1980. Berlin est en effet le lieu où « s'estompent les frontières entre les sphères publique et privée », un espace en marge de la normalité, propice au développement d'un esprit critique, non-conformiste et, compte tenu du statut particulier de la ville, nécessairement «politisé ». Si la personnalité de l'auteur s'ancre dans l'histoire familiale et le rejet du père, il ne fait nul doute que les prédispositions de l'enfant et de l'adolescent, son don de l'observation, sa passion pour la littérature et la « chose politique », sa curiosité de l'autre trouvaient en Berlin la possibilité d'un plein épanouissement. Une tension permanente entre objectivité et subjectivité, entre l'expérience personnelle et collective, de même que le rejet des jugements péremptoires, des attitudes partisanes et de toute idolâtrie confèrent sa spécificité au regard porté sur soi et sur la société environnante, dans un exercice constant du jugement critique et une prise de distance qui passe non pas par le registre de l'accusation et de la condamnation sans appel, mais par le jeu des contrastes : entre Berlin et le reste de l'Allemagne (principalement la RFA) et, si l'on considère les années d'apprentissage, entre une génération de jeunes Berlinois bien décidés à prendre leur vie et leur avenir en main et la génération précédente, celle du refoulement d'un passé récent et des accommodements avec le présent dont le récit fournit de multiples exemples. Dans ce contexte, le discours tenu par Willy Brandt le 5 novembre 1956 marque une césure et fait figure de révélation pour l'adolescent, fasciné par cet orateur d'un type nouveau s'entendant à libérer chez ses auditeurs leur " potentiel démocratique ».

Le long chapitre consacré aux années de formation à la Freie Universität, lieu du développement de l'esprit, au centre de la vie politique, intellectuelle et culturelle berlinoise, et à l'avant-garde des études comparatistes que Peter Szondi allait révolutionner, abolissant les frontières entre la littérature et l'histoire des idées, constitue pour tout lecteur germaniste une formidable source d'information et de stimulation : qu'il s'agisse des controverses qui animent la $F U$ des débuts du 
mouvement étudiant à la veille des années de plomb, de l'enseignement de Szondi, dont Helmut Pillau trace un portrait contrasté, ou de cette constellation de penseurs et d'intellectuels juifs, insufflant à la pensée une certaine radicalité (Szondi - Adorno - Scholem, et à travers eux Benjamin et Celan), dont la fréquentation, la rencontre ou la découverte alimentera désormais la réflexion de l'auteur autour de l'identité juive et de l'identité allemande. Le chapitre consacré au Zentralinstitut für sozialwissenschaftliche Studien DDR-Forschung und Archivbereich, où Helmut Pillau trouve un emploi au début des années 1970 complète le panorama historique, tout en s'inscrivant dans une continuité d'esprit marquée par la curiosité de l'autre Allemagne (présente dès l'enfance), un engagement précoce et jamais démenti pour la réunification dont l'une des conditions était une connaissance précise des évolutions en RDA et, en écho à la politique inaugurée par Willy Brandt, le rejet de l'esprit manichéen de la Guerre Froide.

Le livre se termine par un hommage à l'ami, Claude Vigée, esprit rebelle à la souveraine liberté, grâce auquel l'homme mûr trouvera ce que Berlin n'avait pu lui révéler et éveiller en lui : la force du vivant. Chez ce grand poète et intellectuel juif, il perçoit des échos de Hamann dont les écrits l'avaient fasciné alors qu'il était étudiant et l'exposé (intégré au récit des années de formation) consacré à l'éthique de la langue chez ce penseur marginal présente une approche novatrice et particulièrement stimulante de l'œuvre. « Ce qui dans le contexte théologique apparait comme une certitude de la foi, apparaît [chez Hamann] sur le mode du "peut-être" », une modalité revendiquée par Helmut Pillau, en ce qu'elle seule est susceptible de ménager à l'esprit, tout comme à la ville divisée où a grandi l'auteur, une ouverture sur l'avenir, et qui nous prévient contre la «stérilité du définitif » dont la dénonciation traverse comme un fil rouge les essais et articles de Helmut Pillau.

Andrée Lerousseau

Stephan Pabst, Post-Ost-Moderne. Poetik nach der DDR, Göttingen, Wallstein Verlag, 2016, 483 p.

Mit seiner 2016 veröffentlichten Habilitationsschrift widmet sich Stephan Pabst einem Thema, das die Literaturwissenschaft seit 1989 immer wieder beschäftigte: dem Schreiben von Autoren aus der ehemaligen DDR nach dem Systemwechsel und den Auswirkungen dieser Zäsur auf ihre Werke. Dabei distanziert sich Pabst in seinem einleitenden Überblick über den Forschungsstand gleich zu Beginn von bisherigen Beschreibungsversuchen, die durch ihr rein thematisches Herangehen die formalen Aspekte der literarischen Texte vernachlässigen. Dies gilt sowohl für Arbeiten, die den Korpus der „Wende“- bzw. „Nachwende“Literatur allein dadurch bestimmen, ob ein Werk das historische 
Ereignis „Wende“ darstellt oder nicht, als auch für Arbeiten, die die Literatur nach 1989 auf die Darstellung von Verlusterfahrung reduzieren, auf Therapie und Trauerarbeit. Des Weiteren hinterfragt Pabst das Postulat einer Kontinuität zwischen DDR- und Post-DDR-Literatur, das einerseits dazu dient, literaturgeschichtliche Entwicklungen vereinfachend darzustellen, und andererseits die wissenschaftliche Kontinuität des Untersuchungsgegenstandes DDR-Literatur rechtfertigen soll.

Ausgehend von der Beobachtung, dass bei einigen für die Literatur nach 1989 besonders wichtigen Autoren Veränderungen auf der Ebene der Gattungspoetik festzustellen sind, untersucht Stephan Pabst in seiner Arbeit den möglichen Zusammenhang zwischen dem Systemwechsel von 1989 und der Transformation literarischer Formen. Dieser Zugang soll es ermöglichen, den Einfluss des historischen Ereignisses auf Literatur und Schreiben nicht nur dort aufzuzeigen, wo das Ereignis in der Literatur repräsentiert wird, sondern gerade auch dort, wo es nicht thematisiert wird, eben auf formaler Ebene. Aufgrund der werkgeschichtlichen Perspektive beschränkt sich Pabst auf vier repräsentative und gleichzeitig durch unterschiedliche Generationszugehörigkeit und Gattungsaffinität Vielfalt garantierende Autoren, die das Bild der PostDDR-Literatur maßgeblich prägen: Heiner Müller, Wolfgang Hilbig, Reinhard Jirgl und Durs Grünbein. Weitere Autoren, bei denen ebenfalls nach dem Systemwechsel einsetzende Gattungsveränderungen zu beobachten sind und die Pabsts Ausgangsthese stützen, sind die von ihm erwähnten Jan Faktor und Lutz Seiler, aber auch die Namen von Kathrin Schmidt oder Steffen Mensching ließen sich hinzufügen.

In den ersten vier Kapiteln untersucht Pabst das Werk der Autoren in Hinblick auf die genannten gattungspoetischen Veränderungen sowie auf ihr gewandeltes Verhältnis zur literarischen Moderne. Die Rezeptionsgeschichte der Werke in den bundesrepublikanischen Medien wird in einem fünften Kapitel als inadäquat reflektiert. Jedes der vier Autorenkapitel fokussiert einen bestimmten Aspekt postsozialistischer Literatur, der das Werk der Autoren, aber auch die intellektuelle Situation der damaligen Zeit wesentlich prägt. So liefern die einzelnen Kapitel nicht nur eingehende Text- und Werkanalysen, sondern bieten auch einen gewinnbringenden ideengeschichtlichen Rückblick auf die Zeit nach 1989.

Das Heiner Müller gewidmete erste Kapitel untersucht den Gattungswechsel, in diesem Fall den Übergang vom Drama zu Gedichten, Gesprächen und Interviews, vor dem Hintergrund einer tiefgreifenden Veränderung des Utopieverständnisses im Denken des Autors. Zunächst arbeitet Pabst den Zusammenhang zwischen utopischem Denken und Drama heraus: von den Produktionsstücken der 1960er Jahre bis zu den „postdramatischen“ Stücken am Ende der DDR verfalle die dramatische Form des Werks in dem Maße, in dem der Autor den Glauben 
an eine Realisierung der Utopie in der Geschichte verliert. Während es Müller gelingt, den Gedanken an die Utopie zu retten, indem er sie in der Tradition Ernst Blochs - von der Geschichte ihrer misslungenen Realisierungen trennt und so enthistorisiert, scheint eine Rettung der dramatischen Form nicht mehr möglich. Müller macht den Umbruch von 1989 dafür verantwortlich, dass es ,keine dramatischen Stoffe mehr, keine historischen Konfrontationen [...]" gibt. So verschieben sich nach 1989 die Gattungsakzente in Richtung Gedicht, autobiographische Prosa sowie Gespräche und Interviews, die am Ende ein Drittel des Gesamtwerks ausmachen. Der dabei sich vollziehende Übergang der Sprecherposition zum Subjekt steht sowohl im Widerspruch zum dramatischen Werk, das das Subjekt vor dem unpersönlichen Prozess der Geschichte in den Hintergrund stellte und zur Selbstaufgabe zwang, als auch zu Müllers Verständnis von Autorschaft als Teilhabe an einem kollektiven Prozess. In den Gedichten, Gesprächen und autobiographischen Schriften, die nach 1989 entstanden, spricht der Autor also aus einer Position heraus, die er selbst für problematisch hält. Dabei macht Müller in diesen subjektorientierten Gattungen selbst auf die problematische Stellung des Ichs aufmerksam, und gerade die neuen Inszenierungen dieses Ichs verweisen permanent auf das fehlende dramatische Werk.

Wolfgang Hilbigs im zweiten Kapitel analysiertes Werk zeichnet sich durch mehrere Gattungsverschiebungen aus: der ursprünglich als Lyriker bekannte Autor geht ab Mitte der 1980er Jahre zunehmend zu Prosa und Roman über, von denen er sich später durch die Rückkehr zur Lyrik wieder distanziert. Pabst interpretiert dies als Ergebnis einer Entfremdungsgeschichte, die eng an die Exilsituation des Autors in der Bundesrepublik gebunden ist und die sich auch nach 1989 fortsetzt. Hilbigs letzter Roman, Das Provisorium, liest sich demnach wie eine Selbstverneinung des Romanautors Hilbig. Als stark autobiographisch geprägter Text wird der Roman gerade dort ungenau, wo er den Protagonisten als in der Bundesrepublik erfolglosen Schriftsteller darstellt und die in dieser Zeit entstandenen realen Texte und Erfolge verschweigt. Dieser Widerruf der eigenen, in der Bundesrepublik entstandenen Texte hängt damit zusammen, dass Hilbig in der Literatur kein subversives Potential mehr erkennen kann, so wie es noch in der DDR der Fall war. Auch habe die durch den Literaturbetrieb pervertierte Literatur ihre kritische Funktion, ebenso wie ihre Fähigkeit zur Selbstreflexivität und Selbstkritik eingebüßt. Werkgeschichtlich zeichnen sich besonders Hilbigs Romane Eine Übertragung und „Ich“ durch eine solche Selbstreflexivität aus: einerseits vermag eine metaphorisierte Sprache die Wirklichkeit nicht mehr zu bezeichnen, andererseits entsteht ein Ich-Text, der an der Unterscheidung von Fiktionalität und Autobiographie scheitert. Beide Romane münden in ein Nachdenken 
über den Substanzverlust der Erzählung und kündigen die in Das Provisorium vorhandene radikale Infragestellung der Prosa bereits an. In der Spätphase seines Werks unternimmt der Lyriker Hilbig eine Rückkehr zu den eigenen Wurzeln. Die unter dem Titel Bilder vom Erzählen veröffentlichten Gedichte setzen sich weiter mit der Erfahrung des Substanzverlustes von Erzählen und Literatur auseinander, fokussieren aber nunmehr den Mythos als mögliche Rückkehr zu einem „Urstand des Schreibens“, der ursprünglich derjenige Wolfgang Hilbigs gewesen sein mag.

Das dritte Kapitel behandelt mit Reinhard Jirgl einen Autor, dessen zu DDR-Zeiten unveröffentlichte Texte zunächst im Zeichen einer ausgesprochenen Gattungshybridität standen. Seine experimentellen Romantexte weisen Züge des Dramas auf, wobei Jirgl sowohl von Heiner Müllers postdramatischem Theater als auch von dessen posthumanistischem Denken beeinflusst wurde. Das kritische Potential der frühen Jirglschen Texte bezog sich zunächst auf die Macht- und Gesellschaftsstrukturen in der DDR, deren humanistische Utopien er als im Kern totalitär zu entlarven versuchte. Nach 1989 erweitert Jirgl dann diese Kritik auch auf die westlichen Demokratien und nivelliert in der Tradition von Adornos Kritischer Theorie Systemunterschiede. Während die frühen Texte jegliche Repräsentation verweigern, ändert Jirgl seine poetologischen Prämissen zugunsten eines realistischen Romans, der Unrecht in der Gesellschaft benennt und in medienkritischer Perspektive jegliche Formen der Simulation, der Welt-Fälschung und der Verkünstlichung des Realen denunziert. Dabei kommt zunehmend auch die „Agonie“ der Kritik in den Blick: trotz eines erhöhten Bedarfs an Gesellschaftskritik ist diese kaum noch möglich. Gattungspoetologisch fallen die Einlagerungen essayistischer Passagen in den Romanen auf, welche die zunehmende Reflexivität des Romans der Moderne fortschreiben und gleichzeitig zu einem Höhepunkt bringen: Kritik steht nicht mehr über der Wirklichkeit, sondern ist Teil von ihr und lässt sich nur noch auf der Ebene romanhafter Darstellung verhandeln. Jirgls Gattungsverschiebung von hybriden Formen hin zum realistischen Roman entspricht nicht nur den Erwartungen der Verlage unter veränderten gesellschaftlichen und ökonomischen Verhältnissen. Das Festhalten an einem gesellschaftskritischen Anspruch an die Literatur muss nun einem anderen Geschichtsverständnis Rechnung tragen, das in die Romanform Eingang findet: die abstrakte Reflexion über die Weltgeschichte, die die hybriden Anfänge des Werkes prägte, macht der Geschichte als konkretem historischen Ablauf Platz.

Durs Grünbeins im vierten Kapitel analysiertes Werk vollzieht keinen Gattungswechsel - auch nach dem Epochenumbruch von 1989 bleibt Grünbein Lyriker. Allerdings zeichnet sich seine Dichtung gerade dadurch aus, dass sie Gattungsentwicklungen thematisiert. Dies 
geschieht zunächst in Hinblick auf den Dramatiker Heiner Müller, der Grünbeins literarische Entwicklung stark beeinflusste. In Grünbeins dichterischem Nachruf auf Müller vollzieht dieser den Gattungswechsel des Dramatikers noch einmal nach, wobei Hegels geschichtsphilosophische Gattungstheorie mitschwingt, der zufolge bestimmte Gattungen mit bestimmten Epochen einhergehen und gegebenenfalls auch untergehen: in diesem Falle die von Utopie und Gewalt geprägte Epoche des Sozialismus/Kommunismus und das Drama. Die Tatsache, dass bei Müller auf den Tod des Dramas das Gedicht folgt, rechtfertigt gleichzeitig die Gattung, in der Grünbein selbst schreibt. Unter dem intellektuellen Einfluss der Vertreter der ,Posthistoire“ ist auch für Grünbein nach dem Systemwechsel von 1989 die Geschichte an ihr Ende gelangt. Grünbein selbst positioniert sich in einem ,generalisierten Danach“, jenseits der Geschichte, so dass Transhistorizität und Geschichtsenthobenheit für die poetologische Bestimmung seiner Gedichte bedeutsam werden. Diese Poetologie wird in unterschiedlichen Werkphasen auf verschiedene Weise realisiert: zunächst als ,,posthistorische Physiologie“, die den Körper als das Gegenteil von Geschichte ins Zentrum der dichterischen Reflexion stellt, später durch eine Ausrichtung auf einen „,posthistorischen Klassizismus“, der sich auf die Rezeption antiker, vor allem römischer Vorbilder stützt. Transhistorizität bleibt in Grünbeins Poetologie das Gattungsmerkmal des Gedichts schlechthin.

Im letzten Kapitel der Untersuchung werden die anhand der einzelnen Werkanalysen gewonnenen Erkenntnisse unter dem Gesichtspunkt eines veränderten Verhältnisses zur Moderne zusammengeführt. Während die untersuchten Autoren vor 1989 dezidiert den Anschluss an die Moderne suchten und ihre Literatur damit begründeten (was auch ihren Erfolg nach 1989 garantierte), treten die Modernebezüge vor allem bei Hilbig, Jirgl und Grünbein in den Hintergrund. Hilbig versucht die Literatur der Moderne mit einem Rückgriff auf die Vormoderne, auf den Mythos, zu vermitteln. Jirgls hybride und sprachkritische Anfänge werden in der Romanform moderater, Grünbein distanziert sich Mitte der 1990er Jahre definitiv von der Moderne und ihrem Innovationsdruck. Pabst untersucht in diesem Kapitel auch die Rezeption der genannten Autoren durch den Literaturbetrieb, wobei er zunächst auf eine kommunikative Asymmetrie aufmerksam macht, die dadurch entsteht, dass die Literaturkritik nach 1989 fast ausschließlich westdeutsch geprägt war. Dies führte dazu, dass gerade die westliche Moderne zum Interpretationsmodell der ostdeutschen Literatur erhoben wurde. Während jedoch die radikalen Schreibweisen der Autoren in der DDR einer Abgrenzung von der staatlichen Kulturpolitik dienten (Pabst spricht von „Ausschlusskommunikation“), wurde das moderatere Verhältnis zur Moderne, das die untersuchten Autoren nach 1989 an den Tag legten, Voraussetzung ihrer positiven Rezeption in der 
Bundesrepublik (Moderne als „Anschlusskommunikation“). Autoren, die wie die ehemaligen Protagonisten der DDR-Subkultur an einer radikalen Modernekonzeption festhielten, wurden hingegen nach 1989 weit weniger wahrgenommen. Pabst macht ebenfalls deutlich, inwiefern nach 1989 am Gegenstand der (Post-)DDR-Literatur Debatten über die Geltung, Transformation und das Ende der Moderne ausgetragen wurden, die ihren Ursprung in der alten Bundesrepublik der 1980er Jahre hatten und dazu führten, dass der Moderne-Begriff immer beliebiger eingesetzt wurde.

Zusammenfassend zeigt Pabst auch auf, inwiefern alle behandelten Autoren Kritik als dominante Funktion der Literatur erkennen, ohne diese jedoch als „Engagement“ bzw. politische Intervention zu verstehen. Vor 1989 drückte sich diese Kritik in der ästhetischen Form der Texte und der Sprache aus, und auch nach 1989 suchten alle vier Autoren einen Punkt, von dem aus sich Kritik begründen ließe: die Utopie bei Müller, die Wirklichkeit bei Jirgl, der Mythos bei Hilbig und das Alte bei Grünbein. Dies, so Pabst, lässt sich aber nicht mehr mit Begriffen wie Moderne bzw. Postmoderne fassen und verlangt einen neuen Blick seitens der Literaturkritik, für den die vorliegende Studie eine wichtige Anregung ist.

Carola Hähnel-Mesnard

Robert Straube, Veränderte Landschaften. Landschaftsbilder in Lyrik aus der DDR, Bielefeld, transcript, 2016, $427 \mathrm{~S}$.

Im Zuge des spatial turn, der geographische und kulturelle Räume als paradigmatische Kategorie ins Zentrum kulturwissenschaftlicher Untersuchungen stellt, werden literarische Landschaftsdarstellungen zunehmendnachihremBezugzukonkreten Topographienundgesellschaftlichen Raumvorstellungen hinterfragt. Der Untersuchungsgegenstand der vorliegenden Publikation eignet sich ganz besonders dazu, sozialen und politischen Implikationen von Raumkonstruktionen und deren literarischer Umsetzung nachzugehen. In diesem Sinne fragt Robert Straubes Studie nach spezifischen Formen und Funktionen von Landschaft in der Lyrik der DDR, nach dem besonderen Konstruktionscharakter des ,ästhetischen Raums“ Landschaft und den ihm zugrunde liegenden Raumkonzeptionen und Darstellungstraditionen. Bereits der Titel der Studie verweist auf den besonderen Platz, der der Umgestaltung ländlicher und urbaner Landschaften durch den Menschen in den literarischen Landschaftsdarstellungen zukommt. Die in der SBZ und in der DDR entstehende Lyrik thematisiert keine zeitlos schöne Natur mehr, sondern historische Prozesse und die Einwirkungen des Menschen auf die Landschaft. Diese nicht primär ästhetische Landschaftsauffassung 
dient auch einer Abgrenzung der jungen DDR-Lyrik von den in der Bundesrepublik dominierenden Formen naturmagischer Lyrik.

In einem einführenden Kapitel kontextualisiert Straube den ästhetischen Diskurs über Landschaften, wobei er vor allem drei unterschiedliche Bedeutungsschichten des Begriffs herausarbeitet, die ihm in den folgenden Kapiteln als Analysekategorien dienen: (1) Landschaft als Ausschnitt der Erdoberfläche, der optisch bzw. ästhetisch wahrgenommen werden kann (Landschaft als Ansicht, als Bild); (2) die künstlerische Darstellung dieses empirischen Landschaftserlebnisses als Bild oder Text, die bestimmten ästhetischen, kulturellen und ideologischen Modellen folgt; (3) Landschaft als regionaler und sozialer Zusammenhang, der biographisch und lebensweltlich prägend wirkt.

Das zweite Kapitel widmet sich den Vertretern ,sozialistischer Naturlyrik", die die Entwicklung der DDR-Lyrik von der Nachkriegszeit bis in die 1960er Jahre besonders prägten. Mit Johannes R. Becher und Georg Maurer kommen zwei Autoren zu Wort, die auch eine wichtige kulturpolitische Bedeutung in der DDR hatten: Becher als Minister für Kultur, Maurer als Dozent am 1955 in Leipzig gegründeten Literaturinstitut „Johannes R. Becher“. Beide Autoren vertraten eine traditionalistische Poetik, die sich vor allem durch ihren Rückbezug auf die Weimarer Klassik und das Ideal der Dichtung als Mittel der Bildung und Erziehung des Menschen auszeichnete. Für Becher zeigt Straube auf, inwiefern die Naturlyrik der Nachkriegszeit Modelle seiner Exillyrik fortsetzt, die Landschaften vor allem als Rückzugsräume und Gegenbilder der Zivilisation gestaltet. Die Lyrik der Nachkriegszeit ist weit weniger in der neuen Gegenwart verankert, als man vermuten könnte, sie thematisiert auf eher traditionelle Weise den Gegensatz von Natur und städtischem Leben. Utopische Gehalte wurden in diese Gedichte auf der Grundlage anderer Texte Bechers hineininterpretiert, so dass der Autor dem Anspruch eines Vorreiters der neuen sozialistischen Literatur gerecht werden konnte. Weitaus prägender für die Entwicklung einer eigenen sozialistischen Naturlyrik wirkte Georg Maurer. Durch sein Werk festigte sich das Verständnis vom Natur- und Landschaftsgedicht als einer literarischen Form, die die Beziehung des Menschen zu seiner natürlichen und sozialen Umwelt gestaltet. In Maurers Dichtung bleibt der konkrete Landschaftseindruck oft abstrakt, Natur und Landschaft dienen vielmehr als Folie, um das Verhältnis des Menschen zur Natur vor dem Hintergrund utopischer Vorstellungen darzustellen. Dabei ist Maurers Lyrik ein Bekenntnis zum sozialistischen Staat, gleichzeitig reflektiert sie aber auch allgemein Formen gesellschaftlicher Entfremdung und deren Überwindung im Idealbild einer harmonischen Beziehung zwischen Mensch und Natur.

Mit Peter Huchel und Johannes Bobrowski werden im Anschluss zwei Autoren untersucht, deren Namen man nicht unbedingt mit dem 
Begriff sozialistische Natur-Lyrik in Verbindung bringt, die aber durch ihre ganz spezifischen, an der ästhetischen Moderne orientierten Texte die Anfangsjahre der DDR-Literatur besonders prägten. Beide Autoren verzichten auf den für Becher und Maurer charakteristischen erzieherischen Anspruch der Dichtung ebenso wie auf Bekenntnisse zum neuen Staat, von einem anfänglichen „Pathos des Neubeginns“ bei Huchel einmal abgesehen. Unter Abgrenzung von traditionellen, durch starke Innerlichkeit geprägten Formen der Natur-Lyrik geht es ihnen darum, Geschichte in konkreten räumlichen und sozialen Zusammenhängen darzustellen. Peter Huchel, von 1949-1962 Chefredakteur der Zeitschrift Sinn und Form, stand mit seiner Vorkriegslyrik der naturmagischen Schule nahe, grenzte sich aber bereits durch die verstärkte Beachtung sozialer und gesellschaftlicher Phänomene ab. Seine die Geschichte des brandenburgischen Havellands verarbeitenden Gedichte sind eine Hommage an die dort lebenden einfachen Menschen. Neu an der Nachkriegslyrik ist eine stärkere Bezugnahme auf die Zeitgeschichte, wobei vor dem Hintergrund von Nationalsozialismus und Krieg auch Fragen nach der Bedeutung von Heimat und der möglichen dichterischen Auseinandersetzung mit einem bestimmten Territorium auftreten - Fragen, die Straube in einem Zwischenkapitel noch einmal gesondert diskutiert. Im Laufe der 1950er Jahre wendet sich Huchel mehr und mehr von der Darstellung sozialer Landschaften ab, Landschaft wird nunmehr zur Chiffre individueller, existentieller Fragestellungen. Auch in Johannes Bobrowskis Lyrik erscheinen Natur und Landschaft als Ergebnis einer geschichtlich nachvollziehbaren Einwirkung menschlicher Tätigkeit. Ostpreußische und litauische Landschaften werden zum Ausgangspunkt für eine Auseinandersetzung mit der Geschichte Osteuropas vor dem Hintergrund deutscher Schuld an der Zerstörung dieser sozialen Landschaften. Gleichzeitig versetzt Bobrowski durch die Verwendung des antiken Begriffs Sarmatien konkrete Landschaft ins Symbolische und konstruiert eine imaginäre Heimat und Erinnerungslandschaft, in die seine eigenen Kindheitserinnerungen und Landschaftserfahrungen einfließen.

Bei allen vier Autoren stellt Straube zusammenfassend ein starkes Fortwirken der Gattungstraditionen der Naturlyrik des 19. und frühen 20. Jahrhunderts fest. Zwar wird durch die Thematisierung sozialer, historischer und politischer Aspekte die Distanz zur Naturlyrik der Inneren Emigration gewahrt, doch werden insgesamt eher ländliche Räume und traditionelle Kulturlandschaften evoziert und Praktiken industrieller Naturaneignung ausgeblendet. Neue Perspektiven auf Landschaft finden sich vor allem in der nachfolgenden jüngeren Autorengeneration aus dem Umkreis der „Sächsischen Dichterschule“, die sich dem Thema Landschaft unter neuen Voraussetzungen nähern. In einem weiteren 
umfangreichen Kapitel analysiert Straube das Werk von Wulf Kirsten, Heinz Czechowski und Kito Lorenc.

Wulf Kirsten folgt zunächst seinem poetologischen Anspruch, einen „unberühmten Landstrich in poetischer Rede, also preisend““ vorzuführen. Dabei wird ästhetische Naturbetrachtung durch die Einbeziehung des Sozialen erweitert, indem neben Elementen der historischen Entwicklung einer Landschaft - hier vorwiegend der Gegend um das sächsische Meißen - die Bedeutung gegenwärtiger Prozesse ihrer Veränderung durch menschliche Arbeit einbezogen werden. Auch Kirstens poetische Sprache ist durch die Benutzung eines spezifischen Vokabulars aus dem ländlichen Lebensbereich geprägt. Während die ersten Landschaftsgedichte des Autors seinen eigenen Aussagen zufolge eine affirmative und identifizierende Haltung seinem Land und seiner Gegenwart gegenüber zur Voraussetzung hatten, ist die Lyrik der 1970/1980er Jahre angesichts des Bewusstseins kommender ökologischer Katastrophen von kritischeren Tönen geprägt. Das Subjekt steht den Veränderungen seiner Umwelt ebenso ohnmächtig gegenüber wie dem Geschichtsprozess insgesamt, ursprüngliche Glücksversprechen der Gesellschaft werden in Frage gestellt.

Während die Landschaftsdarstellungen im Werk Wulf Kirstens eine Konstante sind, gilt dies für die Lyrik Czechowskis und Lorencs weniger. Straube zufolge ist Czechowski ein ,Verlegenheitslandschafter", der seine eigentlichen Themen, die Suche nach Wurzeln und Reflexionen über den Verlust des Ichs und der Sprache, auch durch Landschaftsdarstellungen vermittelt. Diese können idyllisierend sein, dem lyrischen Subjekt lediglich als Anhalts- und Ruhepunkt dienen, oder aber als „schöne“" Natur angesichts des Ausmaßes geschichtlicher Zerstörung Abwehrund Schuldreflexe auslösen. Czechowski, der persönlich stark durch die Zerstörung der Stadt Dresden betroffen ist, verbindet in seiner Lyrik ästhetische Wahrnehmung und Geschichtsbewältigung, so dass Landschaften für ihn dort interessant werden, wo in ihnen das kollektive Gedächtnis sichtbar gemacht werden kann. Neben diesen vornehmlich Erinnerungsprozessen gewidmeten Landschaftsgedichten schrieb Czechowski in den 1980er Jahren wie Wulf Kirsten Warngedichte im Zeichen eines ökologischen Krisenbewusstseins. Der sorbische Lyriker Kito Lorenc, der zunächst auf Deutsch und dann zunehmend auf Sorbisch schrieb, bekannte sich in seinem Frühwerk geradezu leidenschaftlich zur DDR, die ihm sein Studium der sorbischen Sprache und Kultur ermöglichte und deren Minderheitenpolitik diesen Kulturraum zu bewahren half. Dank der Erschließung ihrer Kulturgeschichte, ihrer Traditionen und Sprache vermochte Lorenc es, die Landschaft der Oberlausitz literarisch zu gestalten, wobei er zunächst Vergangenheitsverklärung und Fortschrittsoptimismus vermischte. Davon distanzierte er sich später, indem er seine Lyrik eine „Monumentalversion sozialistischer 
Landschaftsgestaltung“ nannte. Ab der zweiten Hälfte der 1970er Jahre distanziert sich Lorenc in seiner deutschsprachigen Lyrik von Landschaftsdarstellungen ebenso wie vom Thema sorbischer Traditionen und wendet sich sprachkritischer und sprachspielerischer Lyrik zu, durch die er die Voraussetzungen von Öffentlichkeit in der DDR hinterfragt.

Spezifisch an den Landschaftsdarstellungen in der Lyrik der DDR ist, so kann zusammenfassend festgehalten werden, dass sie neben ästhetischen Aspekten die soziale, kulturelle und historische Funktion von Landschaft hervorheben. Dabei stieß das von Becher und Maurer initiierte Programm einer sozialistischen Naturlyrik schon früh an seine Grenzen. Von Beginn an standen ihnen mit Huchel und Bobrowski Autoren zur Seite, die den angestrebten Nexus von Landschaftsdarstellung und einer affirmativen Haltung zur „Heimat“ DDR nicht mittrugen. Die jüngere Generation übernahm den sozial- und kulturgeschichtlichen Anspruch an Landschaftsdarstellungen, distanzierte sich aber spätestens seit den 1970er Jahren von den utopischen Glücksversprechen ihres Staates. Straube warnt zu Recht davor, Landschaftsdarstellungen als Metaphern für eine politische Protesthaltung bzw. eine verdeckte Kritik am Staat zu sehen. Über das Thema der Landschaft werde nicht nur die DDR thematisiert, sondern allgemein uneingelöste Fortschrittsversprechen im Zuge der industriellen Entwicklung, wobei die DDR nur als eine zivilisatorische Variante im Kontext einer insgesamt verworfenen Modernisierung erscheint.

Straubes insgesamt lesenswerte und durch zahlreiche textnahe Analysen gestützte Untersuchung endet dennoch etwas unbefriedigend, wenn mit der ,jüngeren“ Autorengeneration auch Lyriker einbezogen werden, für die Landschaft letztendlich nicht im Mittelpunkt steht. Hier wäre es nützlich gewesen, die Zusammenstellung des Korpus noch einmal genauer zu begründen und zu erklären, warum z.B. die Lyrikerin Sarah Kirsch nicht berücksichtigt wurde. Oder Autoren wie Günter Kunert, Wolfgang Hilbig. Und da Straube auch die Produktion der Autoren nach 1989 berücksichtigt, wäre ein Ausblick auf den von Peter Huchel beeinflussten Lyriker Lutz Seiler durchaus lohnenswert gewesen.

Carola Hähnel-Mesnard 